\title{
Polarized Raman Spectra and Infrared Analysis of Vibrational Modes in L-Threonine Crystals
}

\author{
B.L. Silva \\ Departamento de Ciências Exatas, Fundaçâo Universidade Federal de Rondônia \\ 78900-000 Porto Velho-RO, Brazil
}
P.T.C. Freire, F.E.A. Melo, I. Guedes, M.A. Araújo Silva, J. Mendes Filho
Departamento de Física, Universidade Federal do Ceará, 60455-760 Fortaleza-CE, Brazil

A.J.D. Moreno

Departamento de Física, Universidade Federal do Maranhão, 65080-040 São Luis-MA, Brazil

Received July 18, 1997. Revised October 26, 1997

\begin{abstract}
Polarized Raman and infrared spectra of l-threonine crystals have been obtained at room temperature and an assignment of normal modes is proposed based on group theory analysis. The usefulness of these assignments comes from the fact that some modes are related to important vibrations that can show changes under different pressure and temperature conditions.
\end{abstract}

\section{Introduction}

Studies of temperature vibrational spectra of amino acids by Raman and infrared spectroscopies are useful in obtaining information regarding the behavior of normal modes and stability of the structure under changes of external conditionsTmolecular conformationTthe effects of various types of intermolecular forces on vibrational frequencies and $\Gamma$ sometimesT the nature of hydrogen bonding in these biologically fundamental substances. Also $\Gamma$ optically active amino acids contain many highly efficient optical second-harmonic generators and are promising candidates for a great number of applications [1-3].

L-threonine is an important amino acid found in several proteins of human being such as $\gamma$-globulin $\beta$ lactoglobulinThemoglobinTinsulinT silk fibroinTamong others [2]. By the physical point of view the l- threonine investigation is relevant both owing to the possibility to observe the behavior of a system where the hydrogen bond plays a fundamental role [3- 5] and the techno- logical importance of a material which shows a secondharmonic conversion efficiency greater than 1 relative to potassium dihydrogen phosphate [1].

In this paper we present Infrared (I.R.) absorption measurements and polarized Raman scattering results for l-threonine at room temperature. Assignments of some internal modes are also given.

\section{Experimental}

The experiments were performed on monocrystalline samples grown at controlled temperature $\left(40^{\circ} \mathrm{C}\right)$ from acqueous solutions of l- threonine powder. Infrared spectrum between 400 and $4000 \mathrm{~cm}^{-1}$ of l- threonine powder was obtained by mixing l-threonine powder with KBr using a Hartmann \& Braun MB100 spectrometer. The excitation source in the Raman experiments was a $514.5 \mathrm{~nm}$ radiation from an Argon ion laser and the scattered light was analyzed using a Spex

\footnotetext{
*e-mail: tarso@fisica.ufc.br
} 
1402 double monochromator and a Model C 31034-RF photomultiplier from RCA. The samples were placed into an helium closed-cycle refrigeration system where the temperature could be maintained constant to \pm 0.5 $\mathrm{K}$ and temperature measurements were performed with a copper-constantan termocouple. Geometries for the spectra listed in the text and figures follow the usual Porto notation $\mathrm{A}$ (BC)D.

\section{Crystal structure}

The crystal structure and the information about the hydrogen bonds were accurately determined by Shoemaker et al. in an X-ray diffraction study [2]. It was found that the crystal is orthorhombic with four molecules per unit cell $\Gamma$ space group $\mathrm{D}_{2}^{4}\left(P 2_{1} 2_{1} 2_{1}\right)$. Lthreonine has a zwitterion configuration and in the crystal the molecules are held by a three-dimensional network of hydrogen bonds with the amino group forming two hydrogen bonds of lengths 0.290 and $0.280 \mathrm{~nm}$ and the hydroxyl group forming a hydrogen bond of length $0.266 \mathrm{~nm}$. These hydrogen bonds are linked to the carboxyl oxygen atoms: one of the carboxyl hydrogen atoms is hydrogen bonded to a hydroxyl group and the other to two amino groups [2].

In the primitive cell of l-threonine crystal Teach ion has a $\mathrm{C}_{1}$ local site symmetry. The 204 vibrations can be decomposed into the irreducible representations of the factor group $\mathrm{D}_{2}$ as

$$
\Gamma=51\left(A+B_{1}+B_{2}+B_{3}\right)
$$

Among these modes $\Gamma 1 B_{1} \Gamma 1 B_{2}$ and $1 B_{3}$ belong to the acoustic branch and the others are optical modes. All representations are Raman active and the $B_{1}+B_{2}+B_{3}$ are I.R. active.

\section{Assignment}

The infrared spectrum was taken between 400 and $4000 \mathrm{~cm}^{-1}$ and is shown in Fig. 1. In Table 1 we present the tentative assignment of these modes. In order to do the assignment we used the knowledge of some group wavenumbers and some vibrations in amino acid crystals with similar structures as we will discuss below. In Fig. 2 we show the Raman spectra of l-threonine crys- tals at $300 \mathrm{~K}$ for four scattering geometries which display the modes belonging to the four irreducible representations of the factor group $D_{2}: \mathrm{Y}(\mathrm{ZZ}) \mathrm{X}-\mathrm{A} \Gamma \mathrm{Y}(\mathrm{XY}) \mathrm{Z}$ - $B_{1} \Gamma \mathrm{Y}(\mathrm{ZX}) \mathrm{Z}-B_{2}$ and Y(ZY)X - $B_{3}$. The wavenumber range comprises mainly two regions Tone from 0 to 1700 $\mathrm{cm}^{-1}$ and another between 2700 and $3300 \mathrm{~cm}^{-1}$. In the first region the spectra were taken in three different scanning steps and this explain the two discontinuities in the background lines.

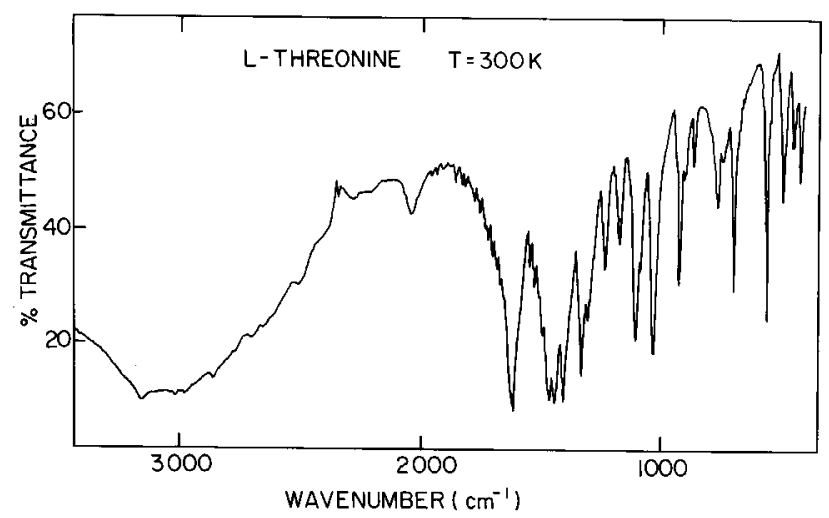

Figure 1. Infrared spectrum of l-threonine crystal at room temperature.

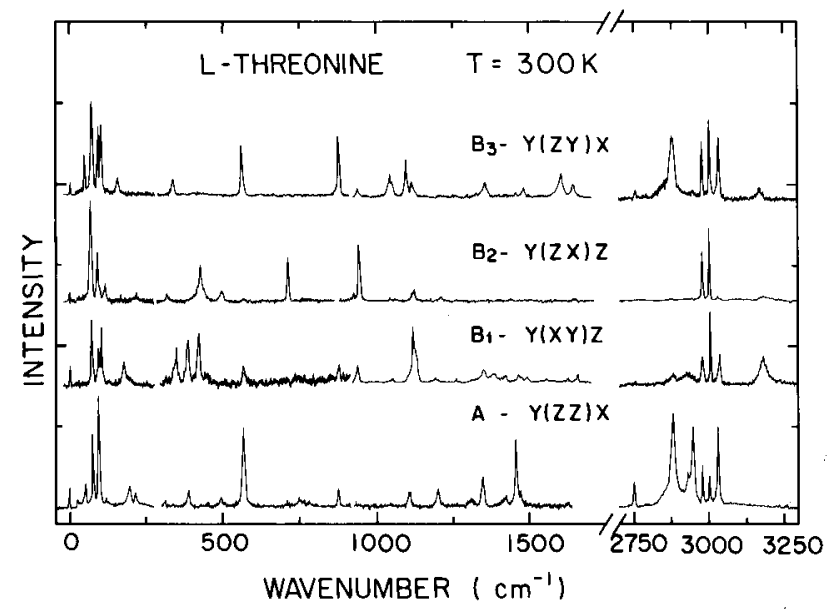

Figure 2. Polarized Raman spectra of l-threonine crystals in the four irreducible representations of D2 factor group at room temperature.

The following discussion refers to Figs. 1 and 2 . The modes with low wavenumbers are related to externalTlibrational and rotational modes and certainly are compressed torsion of $\mathrm{CC}$ structure $\Gamma$ bending of $\mathrm{CCC}$ structure Tamong others [6]. We made a generic assignment - lat. (from lattice) - because it is very difficult to especify exactly any mode in this spectral region; howeverT it is clear that librational and rotational modes are also possible to be found in this spectral region. 
TABLE I :

Assignment of the normal modes of 1-threonine crystal.

\begin{tabular}{|c|c|c|c|c|c|}
\hline $\mathbb{R}$ & $\begin{array}{c}\text { A } \\
Y(Z Z) X\end{array}$ & $\begin{array}{c}\mathrm{B}_{1} \\
\mathrm{Y}(\mathrm{XY}) \mathrm{Z}\end{array}$ & $\begin{array}{c}\mathrm{B}_{2} \\
\mathrm{Y}(\mathrm{ZX}) \mathrm{Y}\end{array}$ & $\begin{array}{l}\mathrm{B}_{3} \\
\mathrm{Y}(\mathrm{ZY}) \mathrm{X}\end{array}$ & Assignment \\
\hline \multirow[t]{12}{*}{$i \cdot-$} & 52 & & & & \\
\hline & 77 & 74 & 68 & 71 & \\
\hline & & & 91 & 93 & \\
\hline & 96 & 96 & 98 & & \\
\hline & & 104 & & 102 & \\
\hline & 120 & & 114 & & \\
\hline & & 175 & & 156 & \\
\hline & 194 & 192 & & & \\
\hline & 215 & & 216 & & \\
\hline & 312 & 315 & 317 & & \\
\hline & & 347 & & 338 & \\
\hline & 388 & 385 & & & \\
\hline 418 & & 421 & 426 & 424 & \\
\hline 447 & 450 & 448 & 448 & & \\
\hline 491 & 493 & & 497 & & $\tau\left(\mathrm{NH}_{3}\right)$ \\
\hline 560 & 570 & 570 & 568 & 564 & $\mathrm{r}\left(\mathrm{CO}_{2}^{-}\right)$ \\
\hline 701 & 709 & & 714 & & $\mathrm{w}\left(\mathrm{CO}_{2}\right)$ \\
\hline 747 & 750 & & 761 & & $\tau(\mathrm{COH})$ \\
\hline 769 & 776 & & 773 & & $\delta\left(\mathrm{CO}_{2}^{-}\right)$ \\
\hline 871 & 878 & 879 & & 878 & $V(\mathrm{CCN})$ \\
\hline 907 & 910 & & & & $v(C C)$ \\
\hline \multirow[t]{2}{*}{932} & 930 & & 925 & & $v(C C)$ \\
\hline & & 940 & 946 & 940 & $v(C C)$ \\
\hline \multirow[t]{2}{*}{1040} & 1032 & & & & $v(\mathrm{CN})$ \\
\hline & 1047 & 1051 & 1044 & 1047 & $V(\mathrm{CN})$ \\
\hline 1093 & & & 1063 & & \\
\hline 1111 & 1108 & & & 1101 & $v_{a}(C C N)$ \\
\hline \multirow[t]{2}{*}{1185} & & 1124 & 1120 & $1117-1124$ & $\mathrm{r}\left(\mathrm{NH}_{3}\right)$ \\
\hline & 1202 & 1197 & 1213 & & $\mathrm{r}\left(\mathrm{NH}_{3}\right)$ \\
\hline 1246 & & & 1263 & 1259 & $\delta(\mathrm{CH})$ \\
\hline 1318 & 1310 & & & & $\delta(\mathrm{CH})$ \\
\hline \multirow[t]{2}{*}{1347} & 1349 & 1350 & & 1355 & $\delta(\mathrm{CH})+$ \\
\hline & & & & & $\delta_{s}\left(\mathrm{CH}_{3}\right)$ \\
\hline 1383 & & 1385 & & & $\delta_{s}\left(\mathrm{CH}_{3}\right)$ \\
\hline 1418 & 1421 & 1423 & & & $\mathrm{~V}_{\mathrm{s}}\left(\mathrm{CO}_{2}\right)$ \\
\hline \multirow[t]{2}{*}{1457} & 1460 & & 1443 & & $\delta_{\mathrm{a}}\left(\mathrm{CH}_{3}\right)$ \\
\hline & 1471 & 1471 & & & $\delta_{s}\left(\mathrm{NH}_{3}^{+}\right)$ \\
\hline \multirow[t]{3}{*}{1480} & & 1491 & & 1483 & $\delta_{s}\left(\mathrm{NH}_{3}{ }^{+}\right)$ \\
\hline & & 1558 & & & \\
\hline & & & & 1606 & $\mathrm{Va}_{\mathrm{a}}\left(\mathrm{CO}_{2}\right)$ \\
\hline $\begin{array}{l}1626 \\
1651\end{array}$ & 1627 & 1629 & & & $\delta_{a}\left(\mathrm{NH}_{3}{ }^{+}\right)$ \\
\hline \multirow{2}{*}{1651} & & 1658 & 1651 & $1641-1647$ & $\delta_{\mathrm{a}}\left(\mathrm{NH}_{3}{ }^{+}\right)$ \\
\hline & 2753 & & & 2757 & \\
\hline \multirow[t]{3}{*}{2873} & $\begin{array}{l}2790 \\
2882\end{array}$ & 2880 & 2886 & 2879 & \\
\hline & $\begin{array}{l}2002 \\
2932\end{array}$ & 2930 & 2000 & & $\mathrm{v}(\mathrm{CH})$ \\
\hline & 2947 & & 2948 & 2950 & $V_{s}\left(\mathrm{NH}_{3}\right)$ \\
\hline 2978 & 2982 & 2981 & 2981 & 2979 & \\
\hline 2998 & 3003 & 3007 & 3004 & 305 & \\
\hline 3026 & 3035 & 3034 & 3034 & 3035 & $V_{a}\left(N_{3}\right)$ \\
\hline 3169 & & 3184 & 3182 & 3170 & $v_{a}\left(\mathrm{NH}_{3}\right)$ \\
\hline
\end{tabular}


Now we begin the tentative assignment of the bands observed with wavenumbers greater than $400 \mathrm{~cm}^{-1}$. To throw light on these assignments knowledge of the I.R. and Raman vibrations in some similar complex molecules is of great importance [7Г8]. In Fig. 1 the band observed at $491 \mathrm{~cm}^{-1}$ was assigned as a torsional mode of $\mathrm{NH}_{3} \mathrm{~T} \tau\left(\mathrm{NH}_{3}\right)$. Such a band was observed with wavenumber of 493 and $497 \mathrm{~cm}^{-1}$ in the $A$ and $\mathrm{B}_{2}$ representations by Raman scattering as is shown in Fig. 2. It is interesting to note that in other materials with amino groups the $\tau\left(\mathrm{NH}_{3}\right)$ is observed near this wavenumber: in a taurine crystal the band was observed around $470-480 \mathrm{~cm}^{-1}[9]$; in alanine crystal the $\tau\left(\mathrm{NH}_{3}\right)$ was observed at $478 \mathrm{~cm}^{-1}[10]$; in a monohydrated l-asparagine crystal $\Gamma$ the band was observed at $429 \mathrm{~cm}^{-1}$ [11] and in a l-asparagine powder the band was observed at $409 \mathrm{~cm}^{-1}$ [12]. Another band associated with torsion of the structure is that observed with a wavenumber of $418 \mathrm{~cm}^{-1}$ which is related to a torsion of the $\mathrm{CC}$ structure $\mathrm{T}$ as was observed in l- asparagine [12].

The band observed with wavenumbers of 560 and $570 \mathrm{~cm}^{-1}$ Trespectively $\Gamma$ in the I.R. and in the Raman spectraTare tentatively assigned as a rocking of $\mathrm{CO}_{2}^{-} \mathrm{Tr}$ $\left(\mathrm{CO}_{2}^{-}\right)$. This is because in l-alanine the $\mathrm{r}\left(\mathrm{CO}_{2}^{-}\right)$is observed at $532 \mathrm{~cm}^{-1}[6]$ and has an intensity greater than the intensity of the $\tau\left(\mathrm{NH}_{3}\right)$ Texactly as occurs with the lthreonine crystal. Also based on the work of Wang and Storms [6] Twe believe that the band with wavenumber of $709 \mathrm{~cm}^{-1}$ in the I.R. and $714 \mathrm{~cm}^{-1}$ in the Raman spectrum is due a wagging vibration of the $\mathrm{CO}_{2}^{-}$structure $\Gamma \mathrm{w}\left(\mathrm{CO}_{2}^{-}\right)$.

A torsion of $\mathrm{COH}$ structure $\Gamma \tau(\mathrm{COH}) \Gamma$ as observed in serine at $752 \mathrm{~cm}^{-1}$ [13] Twas observed by us in the l-treonine around $750-761 \mathrm{~cm}^{-1}$ in Raman spectra and with wavenumber of $747 \mathrm{~cm}^{-1}$ in the I.R. spectrum. The bending of $\mathrm{CO}_{2}^{-} \Gamma \delta\left(\mathrm{CO}_{2}^{-}\right) \Gamma$ was observed by us with wavenumber of $769 \mathrm{~cm}^{-1}$ and $776 \mathrm{~cm}^{-1}$ Tin the I.R. and in the A representation of Raman spectraTrespectively. The same band was observed in l-alanine [6] in both I.R. and Raman at $769 \mathrm{~cm}^{-1}$ and $771 \mathrm{~cm}^{-1} \Gamma$ respectively.

In the $\mathrm{Y}(\mathrm{ZY}) \mathrm{X}$ spectrum of Fig. 2 we observed a very intense band at $878 \mathrm{~cm}^{-1}$ Twhich is observed in the I.R. spectrum at $871 \mathrm{~cm}^{-1}$. This band is related to a stretching band of $\mathrm{CCN}$ structure $\Gamma \nu(\mathrm{CCN})$; this assign- ment is based on the assignment of similar structure in the l-serine and l-cysteine amino acids in water solution $\Gamma$ as observed in Ref. [14]. Bands with wavenumbers of $910 \Gamma 925 \mathrm{~cm}^{-1}$ in the A symmetry and with $940 \mathrm{~cm}^{-1}$ in the $B_{1}$ and $B_{3}$ symmetries are associated to $\mathrm{CC}$ stretching vibrations $\Gamma \nu(\mathrm{CC})$. These assignments are based in the $\nu(\mathrm{CC})$ vibrations of dl-alanine [12]. Bands appearing in the A symmetry with wavenumbers of $1036 \mathrm{~cm}^{-1}$ and $1047 \mathrm{~cm}^{-1}$ are possibly associated to $\nu(\mathrm{CN})$ Ta stretching vibration involving a carbon of the structure and the nitrogen of the amino group. The band observed with wavenumber of $1093 \mathrm{~cm}^{-1}$ in the I.R. spectrum is due to CCN asymmetric stretching vibration as suggested by Diem et al. in a work performed in 1- alanine [15]. The rocking of $\mathrm{NH}_{3}$ structure $\Gamma$ $\mathrm{r}\left(\mathrm{NH}_{3}\right)$ Twas observed in 1- threonine with wavenumbers $1111 \mathrm{~cm}^{-1}$ and $1185 \mathrm{~cm}^{-1}$ in the I.R. spectrum $\Gamma$ showing similar wavenumbers to those observed in l-alanine as studied in ref. [15]. In the Raman spectrum of the $B_{3}-\mathrm{Y}(\mathrm{ZY}) \mathrm{X}$ scattering geometry we observed bands at $1117 \mathrm{~cm}^{-1}$ and $1124 \mathrm{~cm}^{-1}$. All bands observed above are related to the $\alpha_{z y}^{\prime}$ tensor element and are due to polar modes. The band with two peaks at $1117 \mathrm{~cm}^{-1}$ and $1124 \mathrm{~cm}^{-1}$ is possibly due to the additional scattering mechanism [16] which exists as a result of the interaction between polar modes. Since the splitting of the closely spaced components is small $\Gamma$ the effect of the long-range interaction of the internal modes is small. Similar results were observed in the Raman scattering both from $\gamma$-glycine [17] and from l-alanine [6]. In the latter case four pairs of Raman lines were interpreted as the secondary mechanism of scattering involving the polar modes.

Bending vibrations of $\mathrm{CH}$ group $\Gamma(\mathrm{CH})$ Twere found in l- threonine with wavenumbers $1246 \mathrm{~cm}^{-1} \Gamma 1318$ $\mathrm{cm}^{-1}$ and $1347 \mathrm{~cm}^{-1}$ in the I.R. spectrum. We point out that the band corresponding to the I.R. peak at $1246 \mathrm{~cm}^{-1}$ has low intensity in the Raman spectrum observed in $B_{2}\left(1263 \mathrm{~cm}^{-1}\right)$ and $B_{3}\left(1259 \mathrm{~cm}^{-1}\right) \Gamma$ as can be seen in the Fig. 2. Our assignment is consistent to that carried out in l-threonine in water solution [14] and in l-alanine [15]. Bending vibrations of the $\mathrm{CH}_{3}$ group $\Gamma \delta\left(\mathrm{CH}_{3}\right) \Gamma$ were found in l-threonine crystal with wavenumbers $1383 \mathrm{~cm}^{-1}$ and $1457 \mathrm{~cm}^{-1}$ in the I.R. spectrum and with similar values in the Raman 
spectra; once more Tassignment is based on the work of Refs. [14 - 15].

The symmetric stretching of $\mathrm{CO}_{2}^{-}$structure $\Gamma$ $\nu_{s}\left(\mathrm{CO}_{2}^{-}\right) \Gamma$ was found at $1421 \mathrm{~cm}^{-1}$ and $1423 \mathrm{~cm}^{-1}$ in the Raman spectra of $\mathrm{A}$ and $B_{1}$ symmetries. Our assignment is based on the work of both Ref. [14] where such a mode was observed with wavenumber $1409 \mathrm{~cm}^{-1}$ in l-threonine water solution and Ref. [15] where the $\nu_{s}\left(\mathrm{CO}_{2}^{-}\right)$was observed with wavenumber of $1410 \mathrm{~cm}^{-1}$ in l-alanine.

The symmetric bending of the $\mathrm{NH}_{3} \mathrm{\Gamma} \delta_{a}\left(\mathrm{NH}_{3}^{+}\right) \Gamma$ was observed in l-threonine crystals at the wavenumber $1471 \mathrm{~cm}^{-1}$ and $1491 \mathrm{~cm}^{-1}$ in the $\mathrm{B} 1$ symmetry by Raman scattering. This assignment is consistent with the work by Diem et al. [15] $\Gamma$ who observed that mode with wavenumber $1498 \mathrm{~cm}^{-1}$ in l-alanine crystal. The mode observed in the $B_{3}-\mathrm{Y}(\mathrm{ZY}) \mathrm{X}$ scattering geometry with wavenumber $1606 \mathrm{~cm}^{-1}$ was assigned as the asymmetric stretching of $\mathrm{CO}_{2}^{-} \Gamma \nu_{a}\left(\mathrm{CO}_{2}^{-}\right) \Gamma$ and the modes observed with wavenumbers $1641 \mathrm{~cm}^{-1}-1647$ $\mathrm{cm}^{-1}$ were assigned as the asymmetric bending of the $\mathrm{NH}_{3}^{+} \Gamma \delta_{a}\left(\mathrm{NH}_{3}^{+}\right)$. We point out that in l-alanine crystals the $\nu_{a}\left(\mathrm{CO}_{2}^{-}\right)$and $\delta_{a}\left(\mathrm{NH}_{3}^{+}\right)$modes were observed at wavenumbers $1595 \mathrm{~cm}^{-1}$ and $1647 \mathrm{~cm}^{-1} \Gamma$ respectively [6]. Again Twe observed a pair of Raman lines (1641 $\mathrm{cm}^{-1}-1647 \mathrm{~cm}^{-1}$ ) originating from only one vibrational mode as consequence of the interaction between polar modes producing a secondary scattering mechanism.

Finally we discuss the modes with high wavenumbers. These modes are due to the stretching vibrations in both the $\mathrm{NH}_{3}$ and $\mathrm{CH}$ structures. The $\mathrm{CH}$ stretching vibrations $\Gamma \nu(\mathrm{CH}) \Gamma$ were found with wavenumbers $2882 \mathrm{~cm}^{-1}$ and $2932 \mathrm{~cm}^{-1}$ in the A symmetry of the Raman spectrum $\Gamma$ while in the I.R. spectrum the above specified vibration was found with wavenumber 2873 $\mathrm{cm}^{-1}$. This assignment is based mainly on the work of Ref [15] where a meticulous study in l-alanine showed such vibration with wavenumber of $2962 \mathrm{~cm}^{-1}$.

The $\mathrm{NH}_{3}$ stretching vibrations $\Gamma \quad \nu_{s}\left(\mathrm{NH}_{3}\right) \Gamma$ are present in all amino acids and also in other amino substances such as taurine [9]. Among the free $\mathrm{NH}_{3}$ molecule vibrations we known that the symmetric stretching has no degeneracy $\mathrm{C}$ but the asymmetric stretching $\Gamma \nu_{a}\left(\mathrm{NH}_{3}\right) \Gamma$ is doubly degenerate. The $\mathrm{NH}_{3}$ molecule symmetry is $\mathrm{C}_{3 v}$ while the symmetry of the amino group in the crystal environment of the lthreonine is $\mathrm{C}_{1}$. The $\mathrm{C}_{1}$ local site of $\mathrm{NH}_{3}$ is the same in other amino acid crystals. Because of this lowering in symmetry the degeneracy of the asymmetric stretching mode is raised and we can observe two bands corresponding to the $\nu_{a}\left(\mathrm{NH}_{3}\right)$ vibration. For example in the work of Diem et al. [15] the two modes were observed at wavenumbers $3080 \mathrm{~cm}^{-1}$ and $3060 \mathrm{~cm}^{-1}$; in Ref. [18] the two modes are also observed. In a Raman polarized study performed in a monohydrated l-asparagine crystal [11] were observed the two $\nu_{a}\left(\mathrm{NH}_{3}\right)$ modes with wavenumbers $3099 \mathrm{~cm}^{-1}$ and $3115 \mathrm{~cm}^{-1}$; in a Raman study performed in l-asparagine powder it was observed a large band assigned as the asymmetric stretching of $\mathrm{NH}_{3}$ and possibly in such a band the two modes can be found. In the material investigated in the present studyCl-threonine crystal $\Gamma$ we observed the $\nu_{s}\left(\mathrm{NH}_{3}\right)$ with wavenumber $3003 \mathrm{~cm}^{-1}$ for the A symmetry and $2998 \mathrm{~cm}^{-1}$ in the I.R. absorption spectrum. Such a mode was observed with a low intensity in the A symmetry but in the spectrum of the $B_{3}$ symmetry it is observed as the most intense band in the spectrum. One of the $\mathrm{NH}_{3}$ asymmetric stretching vibration was observed in l-threonine crystals by Raman scattering with wavenumber $3034 \mathrm{~cm}^{-1}$ in the $B_{1}$ and $B_{2}$ and wavenumber $3035 \mathrm{~cm}^{-1}$ in the $B_{3}$ and A representations. The other $\mathrm{NH}_{3}$ asymmetric stretching vibration was observed by Raman scattering with wavenumbers between $3170 \mathrm{~cm}^{-1}$ and $3184 \mathrm{~cm}^{-1}$ in the Bs representations $\Gamma$ while it was not observed in the $\mathrm{A}$ representation. Finally stretching of the $\mathrm{NH} 3$ was observed with wavenumbers $3026 \mathrm{~cm}^{-1}$ and $3169 \mathrm{~cm}^{-1}$.

As a conclusion $\Gamma$ polarized Raman spectra in the four irreducible representations of the $\mathrm{D}_{2}$ factor group of the l-threonine crystal were analyzed. As a future work we will investigate amino acid crystals under high hydrostatic pressure and temperature changes in order to investigate eventual phase transitions driven by changes in hydrogen bonds. Structures bonded by ionic bonds are harder than structures bonded by hydrogen bonds. This explainTfor example Twhy the fusion of hydrogen bonded materials occurs at temperatures lower than the temperatures where it is observed the fusion of ionic materials. This suggests that the amino acid 
crystals can show a great number of phase transitions. Hydrostatic pressure is a powerful technique to induce phase transitions in crystals and we believe that with relatively low pressure we can change the distance between the atoms that participate in the hydrogen bond in the amino acid crystals $\Gamma$ and consequently $\Gamma$ we could induce phase transitions $\Gamma$ as was already observed in the monohydrated l-asparagine crystal [19] and in the l-threonine crystal[20].

\section{Acknowledgments}

We are indebt to Prof. L.M.B. Torres from Departamento de Química da Universidade Federal do Maranhão for kindly taking the infrared spectrum and A. J. Melo for drawing the figures. This work had financial support from Fundação Cearense de Apoio à Pesquisa (Funcap) and Conselho Nacional de Desenvolvimento Científico e Tecnológico ( $\mathrm{CNPq})$.

\section{References}

1. K. E. ReickhoffTW. L. PeticolasTScience 147Г610 (1965).

2. D. P. ShoemakerTJ. DonohueTV. Schomaker and R. B. CoreyГJ. Am. Chem. Soc. 72Г2328 (1950).

3. A. Lautié and A. NovakTChem. Phys. Letters 71Г 290 (1980).

4. V. A. Savel'ev and N. D. SokolovTChem. Phys. Letters 34Г281 (1975).

5. M. FalkГJ. Raman Spectrosc. 21Г563 (1990).
6. C. H. Wang and R. D. StormsT J. Chem. Phys. 55Г3291 (1971).

7. L. J. BellamyIThe Infra-red Spectra of Complex

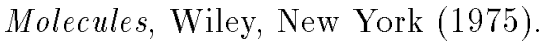

8. G. HerzbergT Infrared and Raman Spectra of Polyatomic Molecules $\Gamma$ Van Nostrand Reinhold N New York (1945).

9. P. T. C. FreireT F. E. A. MeloTJ. Mendes FilhoT J. Raman Spectrosc.27, 507 (1996).

10. S. ForssTJ. Raman Spectrosc. 12Г266 (1982).

11. A. J. D. MorenoTPhD ThesisTCeará Federal UniversityГ1996 (unpublished).

12. K. MachidaГA. Kagayama and Y. SaitoГJ. Raman Spectrosc. 7 Г188 (1978).

13. H. SusiГD. M. Byler and W.V. GerasimowiczГJ. Mol. Struct. 102Г63 (1983).

14. A. R. GargaroTL. D. Barron and L. HechtTJ. Raman Spectrosc. 24Г91 (1993).

15. M. DiemTP.L. PolavarapuTM. OboodiTand L.A. NafieГJ. Am. Chem. Soc. 104T3329 (1982).

16. J. C. DeciusTJ. Chem. Phys. 49Г1387 (1968).

17. R. K. Khanna and P.J. MillerTSpectrochim. Acta A26Г1667 (1970).

18. D. M. Byler and H. Susi Spectrochim. Acta 35AT 1365 (1979).

19. A. J. D. MorenoTP. T. C. FreireT F. E. A. MeloT

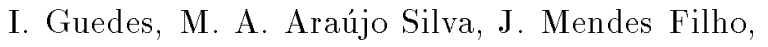
Solid State Commun. 103Г655 (1997).

20. B. L. SilvaГ P. T. C. FreireT J. Mendes FilhoГF. E. A. Melor I. GuedesT M. A. PimentaГM. S. S. Dantas to be published. 